\title{
ANALYSIS OF PERFORMANCE OF FLAT EARTH PHASE REMOVAL METHODS
}

\author{
Keya Desai ${ }^{1}$, Prakruti Joshi ${ }^{1,}{ }^{*}$, Sanid Chirakkal ${ }^{2}$, Deepak Putrevu ${ }^{2}$, Ranendu Ghosh ${ }^{1}$ \\ ${ }^{1}$ Dhirubhai Ambani Institute of Information and Communication Technology, Gujarat, India - \\ (keyadesai97, prakrutijoshi10)@gmail.com, ranendu_ghosh@daiict.ac.in \\ ${ }^{2}$ Microwave Techniques Development Division, SAC, ISRO, Ahmedabad, India - (sanid,dputrevu)@sac.isro.gov.in
}

\section{Commission V, SS: Natural Resource Management}

KEY WORDS: Flat-earth effect, interferometric synthetic aperture (InSAR), Polynomial Fitting, Precise Orbit

\begin{abstract}
:
Interferometric synthetic aperture radar (InSAR) has been widely used in remote sensing field, which can reflect actual topographic trend or possible surface deformation. Due to the orbit attitude influence, the flat-earth phase usually causes the interferogram dense and difficult to be used in further procedures. Before phase unwrapping, interferogram must be flattened to derive accurate topographic or deformation information. In this paper, analysis of performance of two methods of flat-earth removal is done. First method uses imaging geometry and second method uses precise orbital information. Further, 3-degree, 5-degree and 7-degree polynomials are fitted in the method using precise orbital information. Validation is done both visually and empirically using entropy as the evaluation index.
\end{abstract}

\section{INTRODUCTION}

Interferometric synthetic aperture radar (InSAR) has been an important hotspot and widely used in radar imaging area for its high ability to retrieve digital elevation model (DEM) and surface deformation information, which can reflect such information according to the interferometric phase between two or more images [1]. During data processing, phase difference between the scattering elements on the same height level, which is named as flat-earth phase or linear phase, varies because of the spatial geometrical influence and thereby results in the phase offset in the interferogram. This phenomenon is called as flat-earth effect [2]. The phenomenon also showed that the interferogram presented periodic interference fringes. These dense parallel stripes observed in an interferogram caused by flat-earth effect can overshadow the phase variety of topographical changes, and moreover bring much burden for subsequent phase filtering and unwrapping. Therefore, the removal of the flat earth effect is crucial in the InSAR processing [3].

\section{STUDY AREA}

The ancient city of Bam, in southeastern Iran is chosen as the study area. A powerful earthquake struck the city, which destroyed a large part of the city. Three images of the disaster were acquired on 11 June 2003, 3 December 2003 and 7 January 2004 by the ASAR sensor onboard the ENVISAT satellite. Because of the availability of acquisitions before and after the event, it is possible by means of SAR interferometry to highlight the deformations caused by the earthquake with possible millimetre precision.

\section{METHODOLOGY}

\subsection{Using Imaging Geometry}

In this method, geometry between the two satellites during the image survey along with satellite orbit information such as

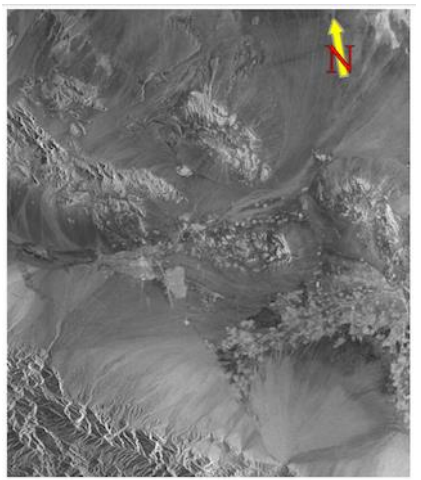

Figure 1. Area under observation

sampling range and range time to first pixel are used to calculate flat earth phase of each pixel [4].

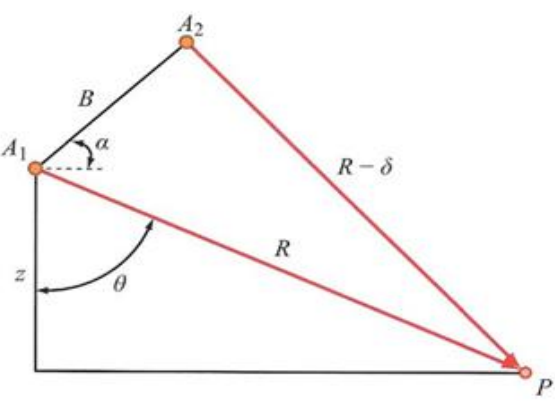

Figure 2. Geometry of flat earth phase

In Fig. 2, A1, A2 represents antenna's positions of two times imaging respectively, the distance between the two antennas is defined as baseline B, $\alpha$ represents the angle between baseline and horizontal surface, $\mathrm{R}$ and $\mathrm{R}-\delta$ are the slant range distances

\footnotetext{
${ }^{*}$ Corresponding Author
} 
between the two satellites and an arbitrary target $\mathrm{P}$ on a terrain respectively, $\theta$ is the look angle and $\mathrm{z}$ is the height of the platform.

The flat-earth phase is given by:

$$
\phi_{f e}=\frac{-4 \pi B}{\lambda R}\left[\sqrt{R^{2}-z_{0}^{2}} \cos \alpha-z_{0} \sin \alpha\right]
$$

The slant range pixel spacing is calculated as:

$$
\Delta r=\frac{c}{2 f_{S}}
$$

Hence, the slant range distance to each pixel can be calculated as:

$$
R=R_{0}+\Delta r * i
$$

where $i$ is in range [0, number of pixels in azimuth direction]

\subsection{Using Precise Orbital Information}

The offset between the master and slave contains the phase information. Thus, it can be calculated on any datum point of the observation region for which the two satellites distance differ, and get the ground phase of the corresponding datum points. The flat earth phase corresponds to path difference as follows:

$$
\phi_{f e}=\frac{-4 \pi}{\lambda}[d(M, p)-d(S, p)]
$$

where M is the Master Satellite Orbit, $\mathrm{S}$ is the Slave Satellite Orbit, $\mathrm{p}$ is a Control point on ground (on WGS84 ellipsoid), $\mathrm{d}$ is the Euclidean distance [5].

The coordinates of $\mathrm{M}$ and $\mathrm{S}$ are known using precise orbits. The exact position of point $\mathrm{P}$ corresponding to a certain line and pixel in the master or slave image can be calculated. Then the flat-earth phase information can be derived from (4). Similarly the phase values of a set of datum points can be used to fit an ndegree polynomial. The function derived then produces the phase value of any arbitrary control point on the interferogram. The flat earth phase for the entire interferogram can be simulated by this method.

A 2-Dimensional polynomial with degree $d$ is defined as:

$$
f(x, y)=\sum_{i=0}^{d} \sum_{j=0}^{i} \alpha_{i-j, j} x^{i-j} y^{j}
$$

Here, $f(x, y)$ can be defined as a 2-D function, which gives the flat-earth phase value at (line, pixel) value as $(\mathrm{x}, \mathrm{y})$.

The number of coefficients (unknown in least square estimation) for a $2 \mathrm{~d}$ polynomial of degree $\mathrm{d}$ is:

$$
\text { No. of coefficients }=\frac{(d+1)^{2}+d+1}{2}
$$

For a 3-degree polynomial, 5-degree and 7-degree polynomial, the number of coefficients or unknowns is 10,21 and 36 respectively. Thus, we need flat earth phase values of 10, 21 and 36 random datum points respectively from the observation region to find out the coefficients of the function. The coefficients of $n$-degree polynomial function are obtained by the solving the set of linear equations represented using matrix and taking its inverse. The function $\mathrm{f}(\mathrm{x}, \mathrm{y})$ obtained after finding the coefficients can now be used to find the accurate flat earth phase by substituting line and pixel values of each data point of the interferogram.

To eliminate the flat earth phase, the simulated flat earth phase obtained using the above methods is multiplied with the complex intensity of each pixel in the interference pattern hence subtracting stimulated flat earth phase from the original phase of the image to obtain flattened interferogram.

\subsection{Entropy}

Entropy is a statistical measure of randomness that can be used to characterize the texture of the input image. It is defined as:

$$
H=-\sum_{i} p_{i} \log p_{i}
$$

where $p$ contains the normalized histogram counts. Raw interferogram has high frequency structured fringes, which are removed on eliminating flat earth phase. On flattening the interferogram, it becomes more unordered and hence the entropy increases. Therefore, we get higher entropy value, for more accurate removal of flat earth phase.

\section{RESULTS AND ANALYSIS}

In this section, sample data is used to validate the two methods described for flat earth removal. For testing, we have chosen the dimension of $10,000 \times 5167$ for ENVISAT data. In the raw interferogram (Fig. 3), we can clearly see the high frequency parallel fringes, which are a result of flat earth effect.

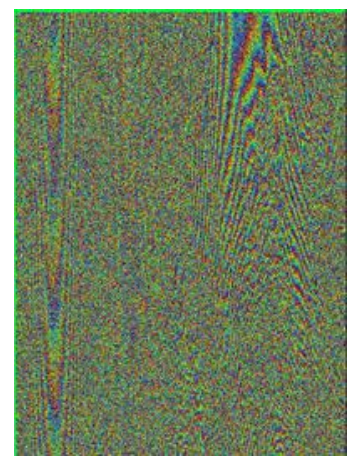

Figure 3. Raw interferogram of ENVISAT data

Using the method of imaging geometry, it can be observed from Fig. 4(a) that high frequency parallel fringes still persist. This method is highly dependent on the parameters such as baseline, look angle and incidence angle whose values may not be known accurately. The calculation errors often cause the inaccuracy in flat-earth phase removal. 


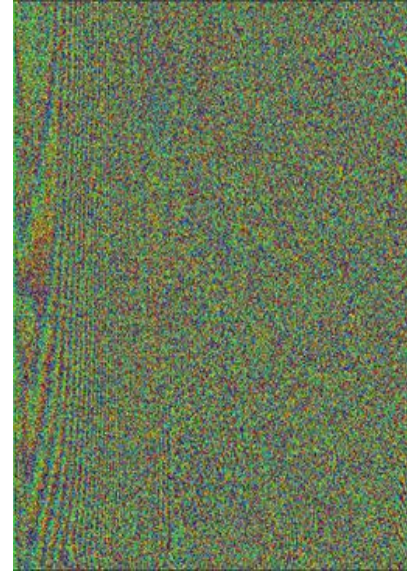

(a)

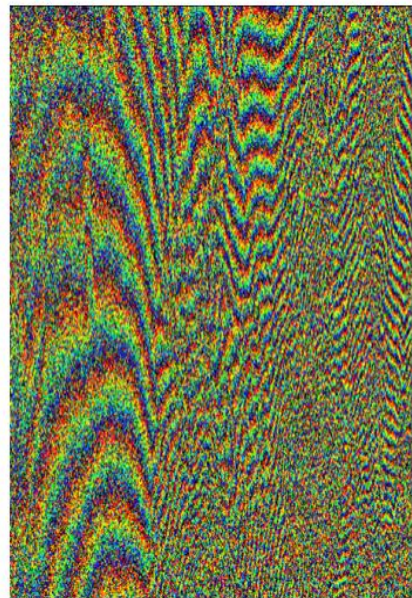

(c)

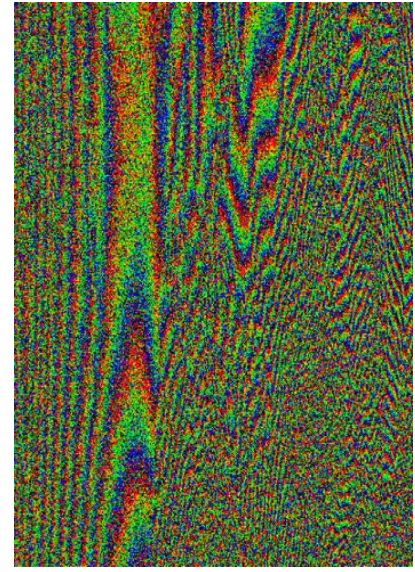

(b)

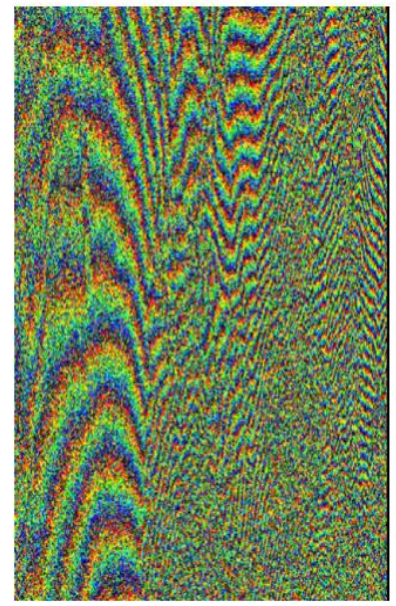

(d)

Figure 4: Interferogram with flat-earth removed using (a) Imaging geometry (b) Fitting a 3-degree polynomial (c)Fitting a 5-degree polynomial (d)Fitting a 7-degree polynomial

For our analysis, in the precise orbital information, we have fitted a 3 -degree polynomial (using 10 ground points), a 5 degree polynomial (using 21 ground points) and a 7-degree polynomial (using 36 ground points). It can be observed from Fig. 4 that using this method, the parallel fringes are almost completely removed. Low frequency fringes are clearly visible in the output. It can be observed that fitting a 5-degree and 7degree polynomial removes more high frequency fringes caused due to flat-earth phase than those removed by fitting a 3-degree polynomial. Fitting a 5-degree and 7-degree polynomial produces similar output.

Fig. 5 shows the comparison of the entropy values using different methods of flat-earth phase removal. We must obtain higher entropy for more accurate removal of flat earth phase. It can be seen from Fig. 5 that on removing the flat-earth phase, entropy value increases. Entropy value increases on going from fitting a 3-degree polynomial to 5-degree polynomial and again shows a slight decrease on fitting a 7 degree polynomial. It can be concluded that the method of fitting a 5-degree polynomial and 7-degree removes more flatearth phase than by fitting a 3-degree polynomial.

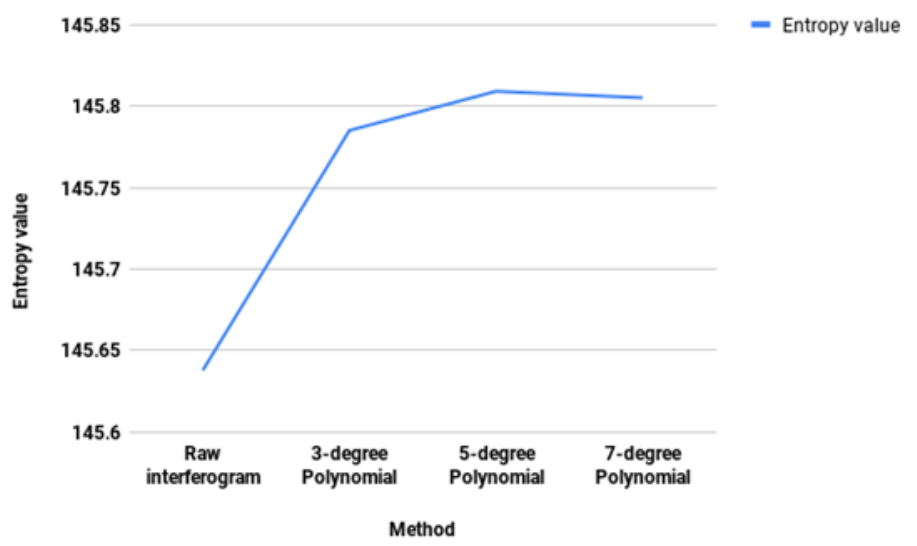

Figure 5: Entropy values of raw interferogram and flattened interferogram using different methods

Furthermore, increasing the degree of polynomial after 5 does not affect the accuracy much and moreover it is much more time consuming. If the phase values at each pixel is calculated directly using Eq. (4), without fitting a polynomial then huge amount of time and computation power is required. Thus it can be inferred that flattening an interferogram by using precise orbital information and fitting a polynomial of degree- 5 is the most optimal method in terms of both accuracy and time efficiency

\section{CONCLUSION}

The basics of flat earth effect removal in InSAR processing are discussed. Performance of two methods of flat-earth phase removal is analysed. The methods are tested on ENVISAT data. The first method using geometry assumes that the parameters remain constant and thus removes the flat earth effect partially and works better on small data. The second method uses the precise orbital information and fits an $n$-degree polynomial to generate the phase. We have fitted 3-degree, 5-degree and 7 degree polynomial. The fifth degree polynomial produces the most accurate output, validated visually and using entropy values and is also time efficient.

\section{REFERENCES}

1. Ai, Bin \& Liu, Kai \& Li, Xia \& Hai Li, Dong. (2008). Flat-earth phase removal algorithm improved with frequency information of interferogram. Proc SPIE. 7147. 46-. 10.1117/12.813247.

2. C. y. Lin, L. Chen and S. q. Ge, "Research on method of flat earth effect removal based on refined local fringe frequency" IET International Radar Conference 2013, Xi'an, 2013, pp. 1-6.

3. Qiming Zeng, Xiaofan Li, Liang Gao, Yihua Liu, "An improvement to flattening in interferometric SAR processing," Proc. SPIE 6200, Remote Sensing of the Environment: 15th National Symposium on Remote Sensing of China, 62000D (9 June 2006);

4. S. Peng, K. He, Y. Wang, J. Lu, J. Deng and H. Xu, A High Accurate Approach for InSAR Flat Earth Effect Removal 2009 International Conference on Measuring Technology and Mechatronics Automation, Zhangjiajie, Hunan, 2009, pp. 742-745.

5. Y. Cao, Z. Fan, Y. Chen, M. Jia, L. Tong and Y. Lu, "Flat earth removal and baseline estimation based on orbit parameters using Radarsat-2 image," 2013 IEEE International Geoscience and Remote Sensing Symposium - IGARSS, Melbourne, VIC, 2013 pp. 346-349. 